\title{
Conformation of Polymers Dispersing Single-Walled Carbon Nanotubes in Water: A Small-Angle Neutron Scattering Study
}

\author{
Yael Dror, ${ }^{\dagger}$ Wim Pyckhout-Hintzen, ${ }^{\ddagger}$ and Yachin Cohen ${ }^{*, \dagger}$ \\ Department of Chemical Engineering, Technion-Israel Institute of Technology, Haifa, Israel 32000, \\ and Institut für Festkörperforschung, Forschungszentrum Jülich GmbH, Germany
}

Received February 20, 2005; Revised Manuscript Received July 1, 2005

\begin{abstract}
Aqueous dispersions of single-walled carbon nanotubes (SWCNT) with polymers were characterized by small-angle neutron scattering (SANS) and cryo-transmission electron microscopy (cryoTEM). Two types of polymers were used: an alternating copolymer of styrene and sodium maleate (PSSty) and gum arabic (GA)-a highly branched polysaccharide. The resulting dispersions were stable and had a homogeneous dark inklike appearance. The dispersions consisted mainly of long isolated nanotubes and thin bundles, as revealed by cryo-TEM. The scattering patterns of both dispersions were fit by a modification of Pedersen's "block copolymer micelle" model, depicting the nanotubes-polymer complex as a thick cylinder hybrid. This hybrid is made of a thin core with radius of about $20 \AA$ containing $\sim 4$ nanotubes surrounded by a thick corona of swollen polymer coils with a radius of gyration of $150-170 \AA$. Long-term stabilization is achieved by the steric barrier provided by the adsorbed polymer coils reinforced by electrostatic repulsion due to charged groups distributed along the polymers.
\end{abstract}

\section{Introduction}

Dispersion of single-walled carbon nanotubes (SWCNT), from the bundled form in which they are often produced, is one of the crucial steps in their processing into useful articles which realize their superior molecular properties. Indeed, much research is currently directed at attaining stable high-quality SWCNT dispersions in a variety of solvents. One route to disperse SWCNT involves functionalization of their wall by reactive groups, which enhance compatibility with the surrounding medium. Alternatively, physical adsorption of dispersants onto the surface of the nanotubes can be effective without local destruction of the nanotube's chemical structure. Within the latter approach, many dispersants have been used, effecting different types of interactions and stabilization mechanisms. Aqueous dispersions have been achieved using small surfactants such as $\mathrm{SDS}^{1,2}$ Triton X-100, ${ }^{2}$ sodium dodecylbenzenesulfonate (NaDDBS), ${ }^{3}$ and pluronic. ${ }^{1,2}$ It was suggested that the surfactants form hemimicelles oriented either parallel or perpendicular to the nanotube axis where the alkyl chains lie along the nanotube's length. ${ }^{3,4}$ Contrarily, it was argued that the formation of hemimicelles on the nanotubes is not energetically favorable due to the high curvature of the nanotubes. Thus, other conformations of the surfactant were reported as a monolayer coating in which the tails are oriented perpendicular to the nanotube $\operatorname{axis}^{5}$ and a structureless adsorbed layer. ${ }^{6}$ Large amphiphilic polymers as poly(vinylpyrrolidone) (PVP) ${ }^{7}$ and natural macromolecules, such as DNA, were used as well. In these cases the structural association between the polymer and the nanotubes was described to be rather robust where the polymer is tightly wrapped around the nanotubes. DNA was also found to be an effective separator between nanotubes having different diameter

\footnotetext{
$\dagger$ Technion-Israel Institute of Technology.

$\doteqdot$ Institut für Festkörperforschung.

* To whom correspondence should be addressed. E-mail yachinc@ tx.technion.ac.il.
}

and chirality according to its specific sequence. ${ }^{8}$ It was shown that a specific 29-residue peptide, which forms an amphiphilic $\alpha$-helix, successfully wraps individual SWCNT in aqueous dispersion. ${ }^{9}$ On the other hand, it was suggested that gum arabic (GA), a highly branched polysaccharide which turned out to be an effective dispersant, forms a more "loose" interaction with the nanotubes, introducing steric repulsion between the adsorbed polymer chains. ${ }^{10}$ It was recently shown that a generic mechanism to disperse isolated nanotubes in any solvent is based on the adsorption of a block copolymer providing selective interaction of the different blocks with the solvent. ${ }^{11}$ In this case it is envisaged that the hydrophobic blocks are tightly adsorbed on the nanotubes surface while the hydrophilic blocks are directed toward the aqueous solution.

The quality of the dispersion in terms of its stability and the degree of debundling has a direct impact on the ability to introduce the nanotubes into a final product in a controllable way, so as to achieve desirable performance. It is especially relevant for fabrication of SWCNT nanocomposites where well-distributed isolated nanotubes are required in order to achieve the significant improvement in mechanical properties predicted by theory. In this case the dispersant has also an important role as the interface binding the nanotubes to the matrix, so that its compatibility with the host matrix is also important. Furthermore, specifically tailored SWCNT/dispersant associations may lead their selfassembly by interaction with functional surfaces. Hence, thorough characterization of the dispersions is of main importance in order to optimize their utilization. Some of the relevant questions are the following: Does the dispersion consist of isolated nanotubes or rather small bundles? How does the dispersant adsorb on the nanotube surface? What degree of coverage is required? And what is the stabilization mechanism?

Scattering methods are powerful techniques to probe structural aspects of nanometric colloidal systems and hence can be advantageously used to investigate carbon nanotube dispersions. To date, few works have exploited 
scattering methods for this purpose. Carbon nanotubes, being thin and long cylindrical molecules with an extremely high aspect ratio and large persistence length, were expected to exhibit a characteristic -1 power law dependence of the scattered intensity on the scattering angle, in well-dispersed conditions. Surprisingly, this has not been observed in most studies, where the observed exponent is closer to -2 . The reason for this discrepancy and its relevance to the structure and quality of the nanotube dispersion is still not totally clear. Schaefer and co-workers studied SWCNT dispersions in polyelectrolyte solutions using a combination of small- and ultrasmall-angle X-ray scattering and light scattering. ${ }^{12,13}$ It was shown that the dispersion consists of branched, ropelike structures of swollen nanotube bundles, rather than isolated nanotubes, thus introducing a power laws of -2.2 in the scattering pattern at different length scales (10-100 $\AA$ and $0.2-28 \mu \mathrm{m})$. Upon extensive sonication, precipitation of carbon aggregates took place. Then, the expected -1 power law gradually appeared at large length scales of 5000-30 $000 \AA$ in the scattering pattern of the remnant dispersion, indicating the existence of isolated nanotubes or straight ropes in the remnant. Dispersion of SWCNT in Triton X-100 also provided a scattering power law of -1.9 at the optimal surfactant concentration as was reported by Wang et al. ${ }^{14}$ It was concluded by the writers that Triton X-100 is not an effective surfactant for dispersing SWCNTs. The authors suggested classifying the dispersions according to the value of the power exponent. As the exponent approaches -1 , the dispersant is more effective. Accordingly, NaDDBS was found to be a very effective dispersant, exhibiting the -1 power law in a limited range of about $310-1600 \AA .{ }^{15}$ At lower angles, the power law was again close to -2 , which was taken to indicate the length scale beyond the nanotube persistence, where the structure resembles a semidilute polymer solution. A detailed analysis of the structural association between the nanotube and the dispersant has not been presented yet.

In this light, the purpose of the current work is to characterize as quantitatively as possible aqueous dispersions of SWCNTs with polymers while attempting to answer the questions presented above. Two different aqueous systems were investigated: (1) gum arabic (GA) and (2) an alternating copolymer of styrene and sodium maleate (PSSty). These polymers were chosen for being large, amphiphilic, and charged molecules, which thus provide steric stabilization reinforced by electrostatic repulsion. However, the polymers differ in their conformation. PSSty is a linear copolymer of alternating hydrophobic and hydrophilic units, while GA is a highly branched polysaccharide. In the sample preparation we tried to avoid harsh treatments such as the use of strong oxidizing acids, long sonication, and strong centrifugation to probe the interaction of the polymers with the nanotube surface which has not been modified chemically, demontrating the true effectiveness of the dispersant at a reasonable concentration.

We pose two main structural issues: the extent to which the nanotubes have bed debundled, i.e., the average number of nanotubes in a dispersed bundle, and the conformation of the polymer around the carbon nanotube, for which the conflicting scenarios are either a tightly bound association ("wrapping") or loose adsorption of swollen polymer coils. The dispersions were characterized by cryo-transmission electron microscopy
Table 1. Composition of the SWCNT Dispersions

\begin{tabular}{cl}
\hline SWCNT/PSSty & \multicolumn{1}{c}{ SWCNT/GA } \\
\hline $0.2 \%(\mathrm{w} / \mathrm{w})$ SWCNTs & $0.2 \%(\mathrm{w} / \mathrm{w})$ SWCNTs \\
$1 \%(\mathrm{w} / \mathrm{w})$ PSSty & $5 \%(\mathrm{w} / \mathrm{w}) \mathrm{GA}$
\end{tabular}

(cryo-TEM) for direct imaging of the vitrified solution and by small-angle neutron scattering (SANS). Scattering at small angles is particularly relevant for comparison of the models for polymer-nanotube interactions. Tight polymer wrapping of the nanotubes is expected to resemble a core-shell cylindrical model, as proposed for surfactant-dispersed nanotubes. ${ }^{6}$ On the other hand, loose adsorption results in a polymernanotube hybrid where the elongated core is decorated by a "hairy corona" of coiled polymers extending into the solution. SANS analysis enabled evaluation of the partial structure factors in order to elucidate the nanotube-polymer interaction.

\section{Experimental Section}

2.1. Materials. Alternate Styrene/Maleic Anhydride Copolymer. This copolymer was a generous gift from Dr. R. Zana (ICS-CRM, Strasburg). The molecular weight of the copolymer was about $50000 \mathrm{Da}$ with undetermined polydispersity. To hydrolyze the anhydride group, the copolymer was boiled with a stoichiometric amount of $\mathrm{NaOH}$ for $2 \mathrm{~h}$, resulting in two carboxylate groups for each repeating units, as shown in the following scheme. The resulting polyelectrolyte ("polysoap") is termed PSSty.

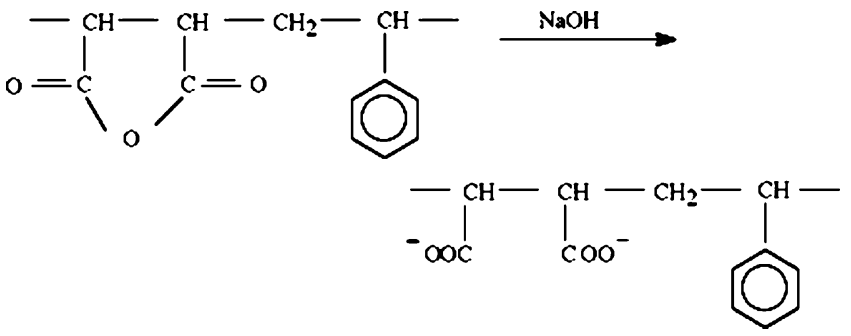

The $\mathrm{pH}$ of the hydrolyzed solution was 9.8. This alternating amphiphilic copolymer has been widely used as a dispersant, ${ }^{16}$ adhesive, binder, ${ }^{17}$ and coating agent. As other alternating amphiphilic copolymers, it has the ability to assemble into a variety of structures in aqueous solution due to intra- as well as intermolecular interactions. ${ }^{18}$ This polysoap was chosen for the dispersion of the nanotubes because of its amphiphilic character, charges along the chain, and the styrene groups that may interact with the nanotube surface via $\pi-\pi$ interaction.

Gum arabic is a natural highly branched polysaccharide extracted from the Acacia Senegal tree. The structure of the GA is rather complex with a characteristic scattering pattern as was extensively discussed in previous report. ${ }^{19}$ GA is known as an excellent emulsifier and stabilizer in food and cosmetic products containing oil-water interfaces. It mostly consists of branched arabinogalactan polysaccharide but also a small amount of arabinogalactan-protein complex responsible for its surface activity. The GA was purchased from Sigma-Aldrich. Solutions of $5 \%(\mathrm{w} / \mathrm{w})$ of GA were prepared in deuterated water. The solutions were gently hand shaken until homogeneous transparent mixtures were achieved. This concentration was found to be the optimal for the dispersion of $0.2 \%$ SWCNTs. ${ }^{10}$

Carbon Nanotubes. SWCNTs synthesized by the electricarc evaporation method were purchased from Carbolex and used as received.

2.2. Dispersions Preparation. The compositions of the different dispersions are given in Table 1. All dispersions were prepared by mixing SWCNT with the solution of the dispersing polymers prepared separately. All dispersions were sonicated 
for $1 \mathrm{~h}$ in a $43 \mathrm{kHz}$ Delta D2000 sonicator, resulting in a homogeneous stable inklike appearance. The dispersions were very stable over months, and only a small amount of solids precipitated over that time. To remove catalyst particles and amorphous carbon, the dispersions were centrifuged for $30 \mathrm{~min}$ at $15000 \mathrm{rpm}$ in a Hettich Mikro 12-24 centrifuge. From weight measurements, the dispersions lost $\sim 50 \mathrm{wt} \%$ of solids by the centrifugation but remained dark.

2.3. Small-Angle Neutron Scattering (SANS). SANS experiments were carried out on the KWS1 and KWS2 diffractometers at the FRJ-2 research reactor in Jülich, Germany. The incident wavelength was $7 \AA$, and the sample aperture was $10 \times 10 \mathrm{~mm}^{2}$. The measurements were done at three collimation/detector setups in order to access the widest scattering vector, $q$, range: C2D2, C8D8, and C20D20 (C stands for collimation distance and D stands for detectorsample distance in meters). Thus, the resulting $q$ range was $0.002-0.152 \AA^{-1}$. The scattering vector, $q$, is given as $q=4 \pi$ $\sin \theta / \lambda$, where $2 \theta$ is the scattering angle and $\lambda$ is the wavelength. The samples were filled in $2 \mathrm{~mm}$ quartz cuvettes. The measured intensity includes the following intensity contributions which have to be subtracted in order to get the net intensity arising from the sample only: empty cell intensity, $I_{\mathrm{EC}}$, and electronic background and $\gamma$ radiation, $I_{\mathrm{BG}}$. The electronic background was measured by placing a piece of boron carbide $\left(\mathrm{B}_{4} \mathrm{C}\right)$ in front of the beam. The net intensity, then, was obtained according to

$$
I_{\mathrm{NET}}(q)=\left(I_{\mathrm{SAMPLE}}-I_{\mathrm{BG}}\right)-\frac{T_{\mathrm{SAMPLE}}}{T_{\mathrm{EC}}}\left(I_{\mathrm{EC}}-I_{\mathrm{BG}}\right)
$$

where $I_{\mathrm{NET}}(q)$ is the net intensity in number of counts in the detector, $I_{\text {SAMPLE }}$ is the measured intensity of the sample, $T_{\mathrm{SAMPLE}}$ and $T_{\mathrm{EC}}$ are the transmissions of the sample and empty cell, respectively, $I_{\mathrm{EC}}$ is the intensity of the empty cell, and $I_{\mathrm{BG}}$ is the intensity of the electronic background. Also, as the detector is a two-dimensional array having $128 \times 128$ channels, the counts were radialy averaged to attain $1 \mathrm{D}$ scattering curves.

Finally, the absolute scattering cross section given in $\left(\mathrm{cm}^{-1}\right)$ was achieved using a secondary standard and according to the following calculation:

$$
\frac{\mathrm{d} \Sigma}{\mathrm{d} \Omega}(q)=\frac{D_{\mathrm{S}} T_{\mathrm{S}}}{D T}\left(\frac{L_{\mathrm{d}, \mathrm{S}}}{L_{\mathrm{d}}}\right)^{2}\left(\frac{(\mathrm{d} \Sigma / \mathrm{d} \Omega)_{\mathrm{S}}}{\left\langle I_{\mathrm{S}}(q)\right\rangle}\right) I_{\mathrm{NET}}(q)
$$

where subscript $\mathrm{S}$ stands for standard and $D, T$, and $L_{\mathrm{d}}$ are the thickness, transmission, and sample-detector distance, respectively. $(\mathrm{d} \Sigma / \mathrm{d} \Omega)_{\mathrm{S}}$ is the known absolute scattering cross section of the standard, and $\left\langle I_{\mathrm{S}}(q)\right\rangle$ is the mean value of the scattering intensity of the standard.

2.4. Cryo-Transmission Electron Microscopy (CryoTEM). Vitrified samples of GA were prepared in a controlled environment vitrification system (CEVS ${ }^{20}$ at a controlled temperature of $25^{\circ} \mathrm{C}$ and $100 \%$ relative humidity. The transfer of the sample to the microscope was done using an Oxford Instruments CT-3500 cryo-specimen holder and transfer system. The samples were investigated using low electron dose imaging and acceleration voltage of $120 \mathrm{kV}$ in a Philips CM120 TEM. Images were recorded with a Gatan MultiScan 791 CCD camera, using the Gatan DigitalMicrograph 3.1 software package.

\section{Small-Angle Scattering Model}

The carbon nanotubes/polymer complex in the dispersion can be depicted as a cylindrical core, composed of a carbon nanotube or a bundle of nanotubes, which is decorated by adsorbed chains. Hence, the model of a "block copolymer micelle", which was written by Pedersen for spherical amphiphilic block copolymers ${ }^{21-23}$ and adapted for cylindrical geometry, ${ }^{24}$ can be followed with appropriate modifications for the present system:

$$
\begin{gathered}
F_{\text {HYB }}=(\Delta \beta)_{\text {core }}{ }^{2} F_{\text {core }}(q)+N_{\text {agg }}(\Delta \beta)_{\text {chain }}{ }^{2} F_{\text {chain }}(q)+ \\
2 N_{\text {agg }}(\Delta \beta)_{\text {core }}(\Delta \beta)_{\text {chain }} S_{\text {core-chain }}(q)+ \\
N_{\text {agg }}\left(N_{\text {agg }}-1\right)(\Delta \beta)_{\text {chain }}{ }^{2} S_{\text {chain-chain }}(q)
\end{gathered}
$$

This expression describes the scattering from the hybrid particle (the "form factor", $F_{\text {HYB }}$ ) as consisting of four terms: the form factor of the cylindrical core, $F_{\text {core }}$, that of the adsorbed chain, $F_{\text {chain, }}$, the cross term between the core and the chain, $S_{\text {core-chain, and the cross term }}$ between the chains in the corona, $S_{\text {chain-chain. }} N_{\text {agg }}$ is the number of chains attached to one cylinder (carbon nanotube or bundle of nanotubes). This differs from the block copolymer model where $N_{\text {agg }}$ represents the number of copolymers aggregated in a micelle. Therefore, the first term is not multiplied by $N_{\text {agg }}^{2}$ as written in the original model, and the third term is multiplied by $2 N_{\text {agg }}$ instead of $2 N_{\text {agg }}{ }^{2} . \Delta \beta_{\text {core }}$ is the excess scattering length of the cylindrical core. It is taken to be composed of $z$ number of nanotubes, each of diameter $r$ and scattering length density of $\rho_{\text {nt }}$. We consider a straight segment of the cylindrical core of length, $L$, which is longer then $q_{\min }^{-1}$, where $q_{\text {min }}$ is the lowest accessible scattering vector. In other words, the model assumes that the hybrid cylinders are rigid with a persistence length larger than $q_{\min }{ }^{-1}$, as consistent with cryo-TEM images shown below. $\Delta \beta_{\text {core }}$ is thus given as

$$
\Delta \beta_{\text {core }}=z \pi r^{2} L\left(\rho_{\mathrm{nt}}-\rho_{\mathrm{s}}\right)=\alpha\left(\rho_{\mathrm{nt}}-\rho_{\mathrm{s}}\right)
$$

The excess scattering length of the polymer chain,

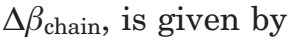

$$
\Delta \beta_{\text {chain }}=V_{\text {chain }}\left(\rho_{\text {chain }}-\rho_{\mathrm{s}}\right)
$$

where $V_{\text {chain }}$ is the volume of the polymer chain and $\rho_{\text {chain }}$ and $\rho_{\mathrm{s}}$ are the scattering length densities of the polymer chain and the solvent, respectively. The form factor of the cylinder core is given by the product of longitudinal and cross-section form factors, $F_{\mathrm{L}}$ and $F_{\mathrm{CS}}$, respectively. Decoupling of longitudinal and cross-section contributions is valid when the cylinder's length is much larger than its cross-section dimension. ${ }^{24}$ Thus, the core form factor is

$$
\begin{aligned}
& F_{\text {core }}(q)=F_{\mathrm{CS}}(q) F_{\mathrm{L}}(q)= \\
& \quad\left(2 J_{1}(q R) /(q R)\right)^{2}\left(2 \operatorname{Si}(q L) /(q L)-4 \sin ^{2}(q L / 2) /\left(q^{2} L^{2}\right)\right)
\end{aligned}
$$

The chain form factor is given by the Debye function for a Gaussian coil:

$$
F_{\text {chain }}(q)=\frac{2(\exp (-x)-1+x)}{x^{2}}
$$

where $x=\left(q R_{\mathrm{g}}\right)^{2}$ and $R_{\mathrm{g}}$ is the radius of gyration (= $b \sqrt{n / 6}, b$ is the length of the monomer, and $n$ is the number of monomers). While eq 7 is strictly valid only for a Gaussian conformation, which evidently is not the case in the studied systems, which are charged, and even branched in the case of GA, this model is still used, however, due to its simplicity while maintaining the main feature of the proposed structure-a coiled polymer loosely adsorbed to the nanotube core. 
The core-chain cross-term is again a decoupled expression; only this time the cross-section term is the product of the amplitudes of each of the entities (core cross section and chains) and the phase term related to the interference between them in a cylindrical geometry:

$$
\begin{aligned}
S_{\text {core-chain }}(q)= & \\
& \psi\left(q R_{\mathrm{g}}\right) \frac{2 J_{1}(q R)}{q R} J_{0}\left[q\left(R+d R_{\mathrm{g}}\right)\right] F_{\mathrm{L}}(q)
\end{aligned}
$$

The chain amplitude $\psi\left(q R_{\mathrm{g}}\right)$ is given by ${ }^{25,26} \psi\left(q R_{\mathrm{g}}\right)=(1$ $\left.-\exp \left(-q^{2} R_{\mathrm{g}}{ }^{2}\right)\right) / q^{2} R_{\mathrm{g}}{ }^{2}$. The parameter $d$ is a necessary factor which takes into account the fact that conformations in which the chain penetrates the core are excluded. Thus, the chains are taken as starting at distance $d R_{\mathrm{g}}$ away from the surface of the core. This factor is close to unity as was demonstrated by simulation and scattering results of copolymer micelles. ${ }^{23}$ Finally, the chain-chain cross-term is

$$
S_{\text {chain-chain }}(q)=\psi^{2}\left(q R_{\mathrm{g}}\right) J_{0}{ }^{2}\left[q\left(R+d R_{\mathrm{g}}\right)\right] F_{\mathrm{L}}(q)
$$

The total scattering cross section is given by

$$
\frac{\mathrm{d} \Sigma}{\mathrm{d} \Omega}\left(\mathrm{cm}^{-1}\right)=n \times 10^{-16} F_{\mathrm{HYB}}\left(A^{2}\right)
$$

where $n$ is the number density of the hybrid particles in particles $/ \mathrm{cm}^{3}$.

\section{Results and Discussion}

The dispersion of SWCNT in PSSty and GA solutions was analyzed by SANS and cryo-TEM. The results are summarized herein where each system is treated separately followed by a comparative discussion between the systems. Before analysis of the specific scattering patterns, we need to address the effect of residual catalyst on the measured SANS patterns. The catalyst can be removed by exhaustive treatment with strongly oxidizing acids. This, however, can modify the nanotube surface chemistry. Extensive cryo-TEM investigations of the raw dispersions have shown that most of the catalyst particles are associated with nanotube bundles and other carbonaceous materials in large objects. As mentioned in the Experimental Section, we used mild centrifugation to minimize the content of the large catalyst and amorphous carbon aggregates, avoiding processes that might introduce functional groups on the nanotubes. The small catalyst particles that remain have a negligible contribution to the scattering pattern in the low range of the measured scattering angles. The expected catalyst scattering at small angles can be estimated as follows. The catalyst volume fraction within the raw solid nanotube material is about $3 \%$. Assuming that about $90 \%$ of the catalyst was removed by centrifugation, and taking a diameter of $200 \AA$ and scattering length density of $9.4 \times 10^{-6} \AA^{-2}$, the expected scattering from catalyst particles at $q \rightarrow 0$ is about 0.015 $\mathrm{cm}^{-1}$, which is insignificant in the small-angle range of interest. However, evaluation of higher resolution details from the SANS patterns at $q>0.02 \AA^{-1}$ is not valid.

4.1. SWCNT + PSSty. Raw scattering patterns of SWCNT dispersions with polysoap (PSSty), as well as that of the bare polymer, are shown in Figure 1 . The dispersion composition is as given in Table 1 , and two different $\mathrm{D}_{2} \mathrm{O} / \mathrm{H}_{2} \mathrm{O}$ mixtures were examined. Three main
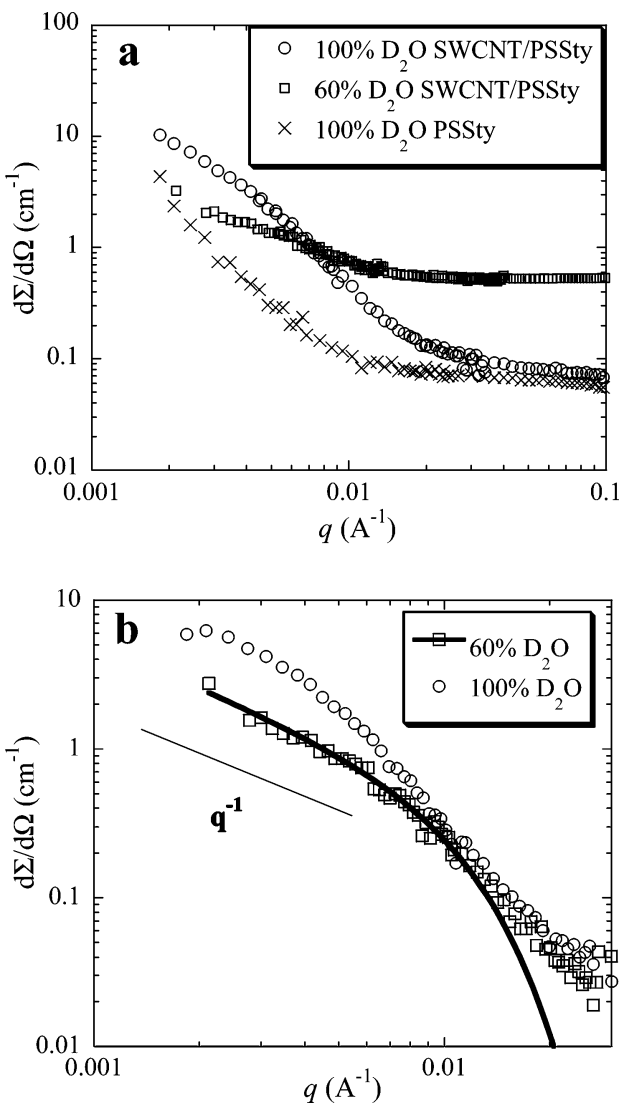

Figure 1. SANS patterns of SWCNT/PSSty dispersions at different $\mathrm{D}_{2} \mathrm{O} / \mathrm{H}_{2} \mathrm{O}$ mixtures: (a) raw data; (b) subtracted data. At $100 \% \mathrm{D}_{2} \mathrm{O}$ scattering from the bare polymer was subtracted from that of the dispersion. At $60 \% \mathrm{D}_{2} \mathrm{O}$ a constant incoherent background was subtracted. The solid line is a fit according to the Guinier approximation for a cylinder, eq 11.

observations can be deduced from these curves. First, the scattering pattern of the dispersion is totally different from that of the bare polymer at the same contrast, in both shape and intensity. The small amount of nanotubes gives rise to excess scattering at the low $q$, indicating that few but large particles are present in the dispersion. Yet, the scattering pattern of the bare polymer itself exhibits also increased intensity at low $q$, more than expected for a polymer of this molecular weight. Second, the $\mathrm{D}_{2} \mathrm{O}$ content of the solvent significantly affects both the shape and the intensity of the scattering pattern. This is a first qualitative indication for heterogeneous hybrid particles consisting of at least two components having different scattering length densities. Third, the $q^{-1}$ power law expected for rodlike particle is not observed at $100 \% \mathrm{D}_{2} \mathrm{O}$. However, this rodlike signature gradually emerges at $60 \% \mathrm{D}_{2} \mathrm{O}$ in the range $0.002-0.006 \AA^{-1}$. As a rough model, the Guinier approximation for cylindrical particles is fit to the data of the $60 \% \mathrm{D}_{2} \mathrm{O}$ dispersion in the low- $q$ range, according to $^{27}$

$$
I=\frac{A}{q} \exp \left(-\frac{R_{\mathrm{c}}^{2} q^{2}}{2}\right)
$$

where $A$ is a prefactor and $R_{\mathrm{c}}$ is the radius of gyration of the cross section. This indicates that cylindrical particles are present in the dispersion and govern the scattering pattern in this $q$ range. The radius of gyration of the cross section, $R_{\mathrm{c}}$, at $60 \% \mathrm{D}_{2} \mathrm{O}$ was found to be 124 

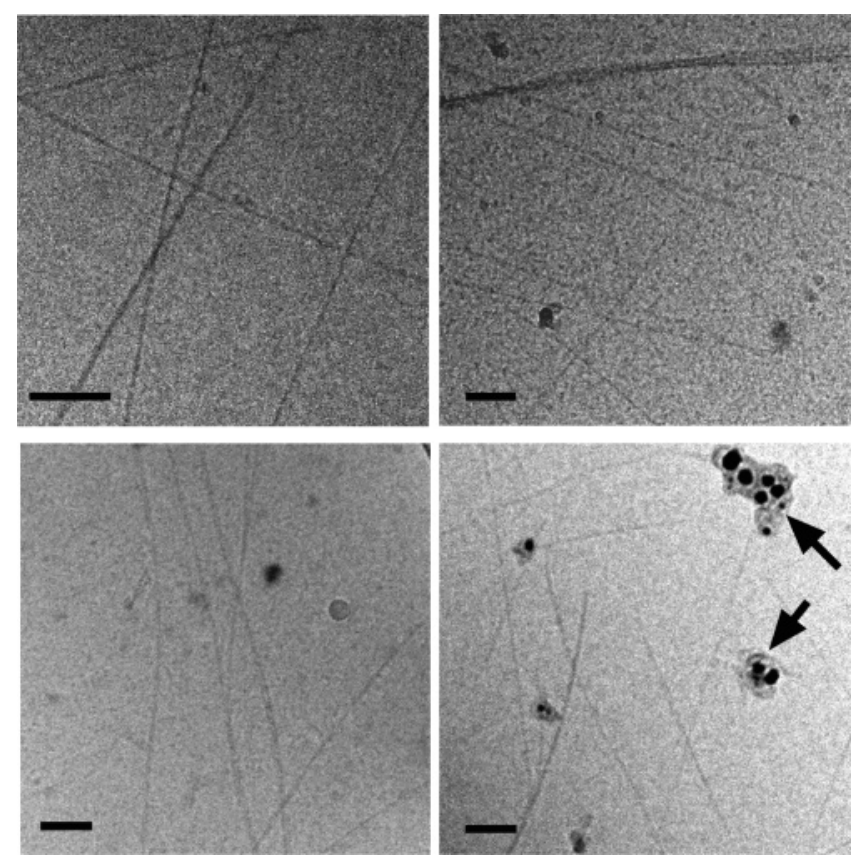

Figure 2. Cryo-TEM micrographs of SWCNT/PSSty dispersion in $100 \% \mathrm{D}_{2} \mathrm{O}$. Arrows indicate catalyst particles surrounded by amorphous carbon, typical of electric-arc-synthesized carbon nanotubes, which were observed occasionally. Bar $=500 \AA$.

$\pm 4 \AA$. This is much larger than expected for an isolated nanotube or a thin bundle.

The scattering intensity is composed of contributions from the SWCNT/polymer complex and unbounded, free polymer. Thus, to treat the complex only, the scattering of the fraction of the unbounded polymer has to be subtracted. The scattering patterns of both the dispersion and the bare polymer at the same contrast coincide at large scattering vectors; hence, the scattering in this range is governed by the free polymer. However, at smaller $q$ the scattering intensity of the bare polymer is significantly lower than that of the dispersion, indicating that it is dominated by the SWCNT/polymer complex. Therefore, the exact fraction of the unbounded polymer to be subtracted is not particularly significant. Indeed, subtraction of all or none of the free polymer scattering has a minor effect on the scattering pattern in the relevant low $q$ range. A similar consideration was discussed by Winey and co-workers. ${ }^{15}$ We decided to subtract the full scattering pattern of the polymer as shown in Figure 1b, suggesting that a relatively small amount of polymer is adsorbed on the nanotubes' surface. For the measurements in $60 \% \mathrm{D}_{2} \mathrm{O}$, a constant incoherent background was subtracted from the scattering of the SWCNT/polymer dispersion, as no significant scattering above this background level was observed in scattering measurements of the bare polymer solutions. Because of these uncertainties in the background subtraction, and the effect of residual catalyst discussed above, evaluation of higher resolution details from the SANS patterns at $q>0.02 \AA^{-1}$ is not valid.

Cryo-TEM micrographs of the aqueous SWCNT/ PSSTY dispersion are presented in Figure 2. Long and thin rodlike features of isolated and very thin bundles of carbon nanotubes are observed. The diameter of the threads ranges from 1 to $5 \mathrm{~nm}$, and the length, which is not entirely seen in the micrographs, is not less than $500 \mathrm{~nm}$. Furthermore, the images do not show any significant bending of the nanotubes in the observed length, indicating a high persistence length. This suggests that if these nanotubes or thin bundles were to scatter as bare entities, a $q^{-1}$ power law is expected in the $q$ range relevant to Figure 1. Evidently, this is not the observation in Figure $1 b$.

In view of the foregoing discussion, it is clear that a structural model for this system must reconcile the cryoTEM observations of long nanotube bundles of rather thin lateral dimensions with the scattering patterns indicating large ones. We therefore applied a modification of the "block copolymer micelle" model by Pedersen (eq 3). As described above, this model describes a cylindrical core formed by a nanotube or a thin bundle, decorated with large polymer coils loosely adsorbed to the nanotubes' surface. The fit parameters are summarized in Table 2, and the curve fitting is presented in Figure 3. The values of the fit parameters are reasonable, considering the physical properties of the constituents. The picture revealed by the values of the fit parameters is of a thick rodlike entity made of a thin and long cylindrical core having a radius of about $20 \AA$. The core is surrounded by a thick water-swollen polymeric corona with a large radius of gyration, about 150 $\AA$. The core may thus be a thin bundle of nanotubes with an average of about 4 (see Table 2). The nanotube core imposes correlations between the adsorbed polymer coils along the cylinder axis. The swollen polymer coils adsorbed on the nanotube impose a large cross-section radius on the composite hybrid. The overall cylindrical structure of the nanotube/polymer complex is presented schematically in Figure 4, noting the relevant sizes. This description explains why the $q^{-1}$ power law cannot be observed in the measured $q$ range, as would have been exhibited by a thinner hybrid. Polydispersity of core diameters, which surely exists, is not significant in the scattering pattern, since the core is only about $10 \%$ of the entire cross section. On the other hand, the model is most sensitive to the structural parameters related to the polymer, i.e., $R_{\mathrm{g}}$ and $N_{\mathrm{agg}}$, as can be seen by the error values in Table 2 . Small deviations in $R_{\mathrm{g}}$ or $N_{\mathrm{agg}}$ immediately affect the curvature of scattering pattern in the concerned range. The coverage of the core by the polymer is determined by $N_{\text {agg }}$ where the number of polymer chains per length of the core ( $N_{\text {agg }} /$ length $)$ is about 5 chains per $200 \AA$. The description presented above is also in line with the cryo-TEM images, since only the nanotube core has sufficient contrast to be observed, while the attached polymer is not seen. It should be noted that PSSty tends to form vesicles and flat objects in aqueous solution by self-assembly, as was shown by cryo-TEM imaging of the bare polymer solution, reported elsewhere. ${ }^{28}$ Cryo-TEM images showing the dispersed nanotubes did not show polysoap vesicles or other assembled structures.

The number density, $n$, of representative hybrid cylinders (5000 A in length) per unit volume, is a scaling parameter which does not affect the curvature of the pattern. Its fitted value is equivalent to a SWCNT weight fraction of about $0.05 \%$ if an average bundle of three nanotubes is considered. The initial concentration of the raw carbonaceous material in the dispersion preparation was $0.2 \mathrm{wt} \%$. The discrepancy may be rationalized by taking into account that precipitation of catalyst particles, amorphous carbon, and large bundles took place during centrifugation (in total amount of $\sim 50$ wt \%). Furthermore, the value of $n$ is inversely related to the polymer-solvent density difference. Thus, 
Table 2. Model Fit Parameters for SWCNT/PSSty Dispersions in $100 \%$ and $60 \% \mathrm{D}_{2} \mathrm{O}$

\begin{tabular}{|c|c|c|c|c|}
\hline \multirow[b]{2}{*}{ parameter } & \multicolumn{2}{|c|}{ dispersion in $100 \% \mathrm{D}_{2} \mathrm{O}$} & \multicolumn{2}{|c|}{ dispersion in $60 \% \mathrm{D}_{2} \mathrm{O}$} \\
\hline & fitted value & error/sensitivity & fitted value & error/sensitivity \\
\hline core radius $R^{a}$ & $20 \AA$ & $\pm 10 \AA$ & $20 \AA$ & $\pm 10 \AA$ \\
\hline polymer radius of gyration, $R_{\mathrm{g}}$ & $150 \AA$ & $\pm 10 \AA$ & $110 \AA$ & $\pm 5 \AA$ \\
\hline unit length $L^{b}$ & $5000 \AA$ & & $5000 \AA$ & \\
\hline chains/length $N_{\text {agg }}$ & 120 & \pm 5 & 210 & \pm 10 \\
\hline number density of hybrid units $n$ & $1.5 \times 10^{14} \mathrm{~cm}^{-3}$ & $\pm 0.1 \times 10^{14}$ & $1.5 \times 10^{14} \mathrm{~cm}^{-3}$ & $\pm 0.1 \times 10^{14}$ \\
\hline$\Delta \rho_{\text {core }}{ }^{c}$ & $-1.5 \times 10^{-6} \AA^{-2}$ & & $1.3 \times 10^{-6} \AA^{-2}$ & \\
\hline$\Delta \rho_{\text {chain }}{ }^{d}$ & $-4.1 \times 10^{-6} \AA^{-2}$ & & $-1.3 \times 10^{-6} \AA^{-2}$ & \\
\hline chain-core exclusion factor $d$ & 0.7 & \pm 0.1 & 0.7 & \pm 0.1 \\
\hline
\end{tabular}

${ }^{a} z$, the number of nanotubes in the core, calculated from the value of $\mathrm{R}$, is about 4 , assuming that the effective radius of individual nanotube is $10 \AA .{ }^{b}$ An arbitrary unit length for the hybrid entity. ${ }^{c}$ The scattering length density of the nanotubes $\rho_{\mathrm{nt}}=4.9 \times 10^{-6} \AA^{-2}$ was taken according to Zhou, ${ }^{15}$ and the scattering length densities of the solvents, $\rho_{\mathrm{s}}$, are $6.4 \times 10^{-6}$ and $3.6 \times 10^{-6} \AA^{-2}$ for $100 \%$ and $60 \%$ $\mathrm{D}_{2} \mathrm{O}$, respectively. ${ }^{d} \Delta \beta_{\text {chain }}$ was calculated according to eq 5 using $V_{\text {chain }}=64.7 \times 10^{3} \AA^{3}$ and $\rho_{\text {chain }}=2.33 \times 10^{-6} \AA^{-2}$ as calculated from the chain density of 1.28 , and chain $M_{\mathrm{w}} \sim 50000$. We could not measured the scattering length density of the bare polymer by matching the solvent due to the high level of incoherent scattering.

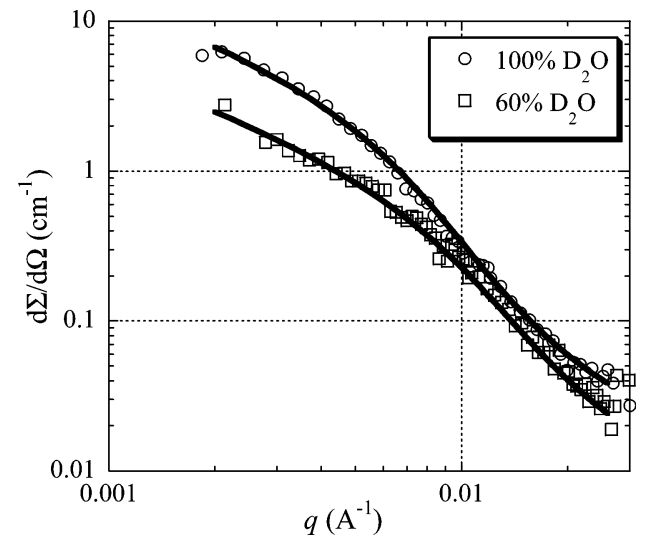

Figure 3. SANS patterns of the SWCNT/PSSty dispersions after subtraction of the polymer solution scattering. Solid lines are the model fit to eq 3 .

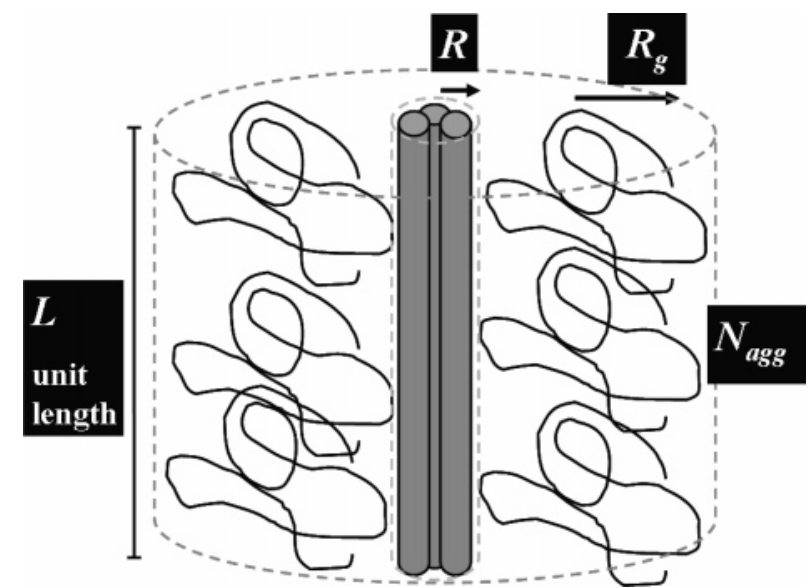

Figure 4. Scheme of the nanotube/polymer complex forming a large hybrid cylinder with a thin core and a thick corona composed of loosely adsorbed polymer coils. The core and corona thicknesses are not drawn to scale.

if water molecules are tightly associated with the polymer, as suggested by the modeling of Whitehead, ${ }^{29}$ the $n$ value is expected to increase due to the decreased difference between the polymer and solvent scattering length densities.

To elucidate the partial contribution of each element of the model to the overall scattering pattern at $100 \%$ $\mathrm{D}_{2} \mathrm{O}$, they are plotted separately in Figure 5 using the calculated and fit values of Table 2. Clearly, the dominant contributions at the low $q$ range are the crossterms, $S_{\text {core-chain }}$ and $S_{\text {chain-chain, which describe the }}$

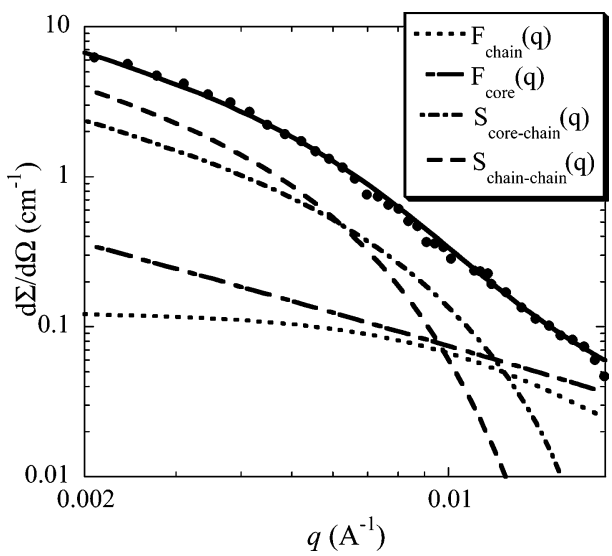

Figure 5. Partial contributions of each term of model in eq 3 to the overall experimental scattering pattern of SWCNT/ PSSty at $100 \% \mathrm{D}_{2} \mathrm{O}$. The functions are calculated using the values given in Table 2 . The continuous line is the summation of the four contributions.

correlation between the core and the chains in the corona and between the chains within the cylindrical corona, respectively. In these terms (eqs 8 and 9) the large cylindrical feature is being expressed. On the other hand, the individual uncorrelated form factors of the thin cylindrical core and the polymer chain are insignificant at this low $q$ range. They become more important at the larger $q$ range, exhibiting the fine curvature of the scattering pattern tail. For full interpretation of the scattering pattern, all expressions need to be taken into account.

Further support for this model is gained by the fact that it also fits the pattern of the dispersion in $60 \% \mathrm{D}_{2} \mathrm{O}$ prepared separately, with only slight differences (Figure 3 ). The excess scattering lengths, $\Delta \beta_{\text {core }}$ and $\Delta \beta_{\text {chain, were }}$ calculated for $60 \% \mathrm{D}_{2} \mathrm{O}$ using the same values of $\rho_{\text {nt }}$ and $\rho_{\text {chain }}$ as in $100 \% \mathrm{D}_{2} \mathrm{O}$ (see note below Table 2 ), but there is a high degree of uncertainty concerning these values since they could not be measured directly. The decrease in the intensity of the corona contribution $\left(\Delta \beta_{\text {chain }}\right)$, combined with the decreased radius of gyration in $60 \%$ $\mathrm{D}_{2} \mathrm{O}$, slightly reveals a -1 power law at low $q$ but not beyond $0.008 \AA^{-1}$. It is not clear yet whether the decreased value of $R_{\mathrm{g}}$ in this fit is attributed to the different $\mathrm{D}_{2} \mathrm{O} / \mathrm{H}_{2} \mathrm{O}$ composition. Further investigations are required to clarify this result.

4.2. SWCNT + GA. The second system investigated is a dispersion of SWCNT in aqueous GA solution, in the composition given in Table 1 . The scattering patterns of the nanotube dispersion and bare GA in two 

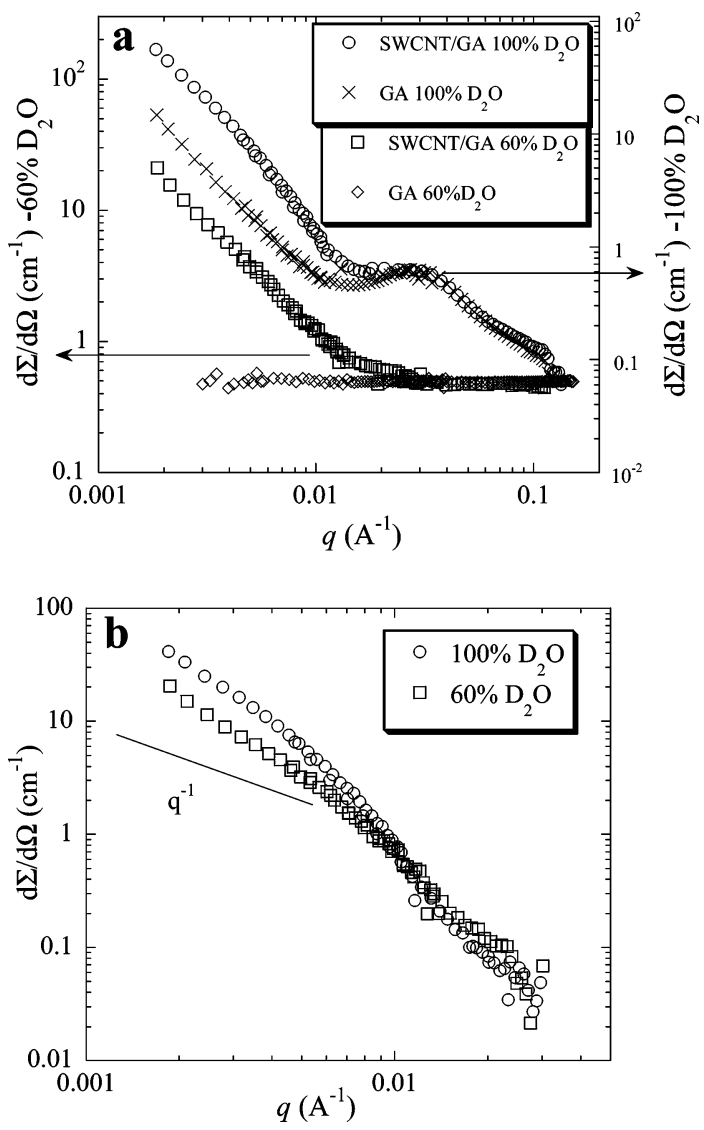

Figure 6. (a) Raw scattering patterns of SWCNT/GA dispersions and bare GA. (b) Subtracted curves.

different $\mathrm{D}_{2} \mathrm{O} / \mathrm{H}_{2} \mathrm{O}$ mixtures are shown in Figure 6a. In contrast to the previous system, here the polymer is highly branched with a rather complex chemical structure and shape. ${ }^{30}$ The scattering pattern of GA is characterized by a peak, attributed to intercorrelation between spheroidal "aggregates". A detailed scattering study of the GA solutions is presented elsewhere. ${ }^{19}$ As shown in Figure 4a, the small amount of nanotubes $(0.2 \% \mathrm{w} / \mathrm{w})$ causes a significant increase in the intensity at the low $q$ range, whereas at larger $q$ the scattering patterns of both the dispersion and the GA overlap. Apparently, in this range the free GA dominates the scattering. This is even more pronounced at $60 \% \mathrm{D}_{2} \mathrm{O}$ where the GA scattering is low and approximately constant, indicating nearly matched contrast. Scattering from the bare GA was subtracted from that of the dispersion, assuming again that most of the polymer is unbounded. The subtracted curves are presented in Figure $6 \mathrm{~b}$. Once again, no sign of a -1 power law is observed.

Cryo-TEM micrographs of the aqueous SWCNT/GA dispersion are presented in Figure 7. As in the previous system, long and thin rodlike features of isolated and very thin bundles are observed. At the bottom image, recorded in large under focus, the nanotubes are less clearly visualized, but the spheroidal "aggregate" character of GA can be easily observed as was reported elsewhere. ${ }^{19}$

The scattering from the SWCNT/GA dispersions was also analyzed using the model of eq 3 . Surprisingly, the model fit the data well, as in the previous system, although the dispersing polymer is totally different. The results of the model fit are presented in Table 3 and Figure 8 . The fit parameters point out the same general
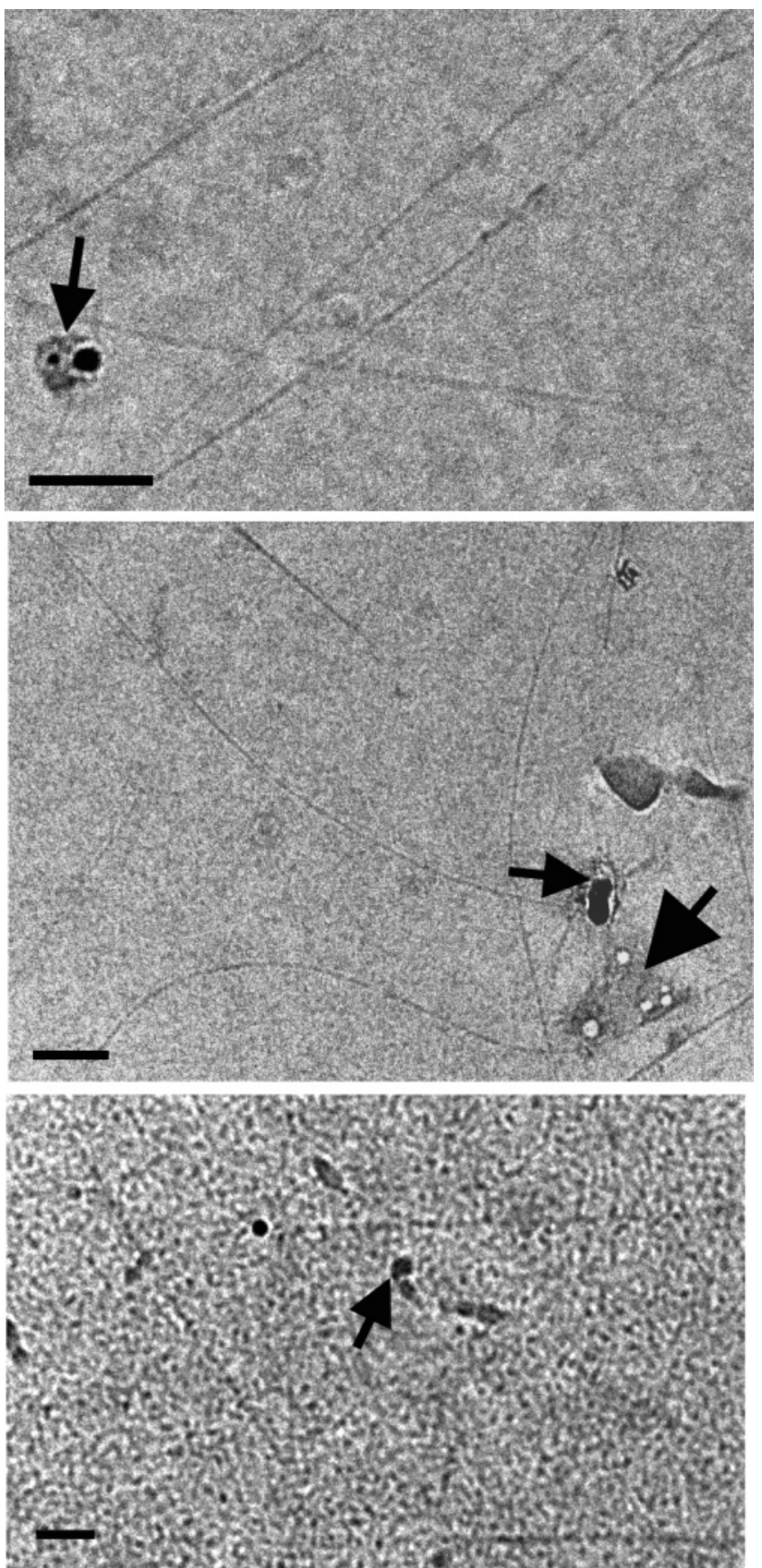

Figure 7. Cryo-TEM micrographs of SWCNT/GA dispersion. Thin arrows indicate catalysts particles surrounded by amorphous carbon. The thick arrow in the intermediate image indicates holes. Bar $=500 \mathrm{~A}$. The bottom image was recorded at under focus condition visualizing the spheroidal "aggregate" character of GA.

view and tendencies. Once again the dispersion consists of thick cylindrical polymer-nanotube hybrids having a thin nanotube core surrounded by large polymer coils. As GA is not a linear polymer, the Debye function may not be the best presentation of its form factor. As before, we use this simple polymer form factor to represent the general view of a solvent-swollen coiled conformation of the polymer surrounding the nanotube. It is interesting to note that although $R_{\mathrm{g}}$ of GA is higher than that of PSSty, $N_{\text {agg }}$ is more than double its value for PSSty, i.e., more than twice the number of molecules covers the same nanotube length. This may be rationalized since GA is a highly branched structure and thus is 
Table 3. Model Fit Parameters for SWCNT/GA Dispersions in $100 \%$ and $60 \% \mathrm{D}_{2} \mathrm{O}$

\begin{tabular}{|c|c|c|c|c|}
\hline \multirow[b]{2}{*}{ parameter } & \multicolumn{2}{|c|}{ dispersion in $100 \% \mathrm{D}_{2} \mathrm{O}$} & \multicolumn{2}{|c|}{ dispersion in $60 \% \mathrm{D}_{2} \mathrm{O}$} \\
\hline & fitted value & error/sensitivity & fitted value & error/sensitivity \\
\hline core radius $R^{a}$ & $20 \AA$ & $\pm 10 \AA$ & $20 \AA$ & $\pm 10 \AA$ \\
\hline $\begin{array}{l}\text { polymer radius of gyration, } R_{\mathrm{g}} \\
\text { unit length } L^{b}\end{array}$ & $\begin{array}{l}170 \AA \\
5000 \AA\end{array}$ & $\pm 10 \AA$ & $\begin{array}{l}170 \AA \\
5000 \AA\end{array}$ & $\pm 10 \AA$ \\
\hline chains/length $N_{\text {agg }}$ & 285 & \pm 15 & 285 & \pm 15 \\
\hline $\begin{array}{l}\text { number density of hybrid units } n \\
\Delta \rho_{\text {core }}{ }^{c}\end{array}$ & $\begin{array}{l}3 \times 10^{14} \mathrm{~cm}^{-3} \\
-1.25 \times 10^{-6} \AA^{2}\end{array}$ & $\pm 0.2 \times 10^{14}$ & $\begin{array}{l}4 \times 10^{14} \mathrm{~cm}^{-3} \\
1.55 \times 10^{-6} \AA^{2}\end{array}$ & $\pm 0.2 \times 10^{14}$ \\
\hline$\Delta \rho_{\text {chain }}{ }^{d}$ & -0.19 & \pm 0.01 & 0.1002 & \pm 0.0022 \\
\hline chain-core exclusion factor $d$ & 0.7 & \pm 0.05 & 0.7 & \pm 0.05 \\
\hline
\end{tabular}

${ }^{a}$ See note in Table $2 .^{b}$ See note in Table 2. ${ }^{c}$ The scattering length density of the nanotubes is $5.15 \times 10^{-6} \AA^{-2} .^{d}$ These values are fit results. No evaluation in terms of $\rho_{\text {chain }}$ and $V_{\text {chain }}$ could be done since the chemical structure is not totally known.

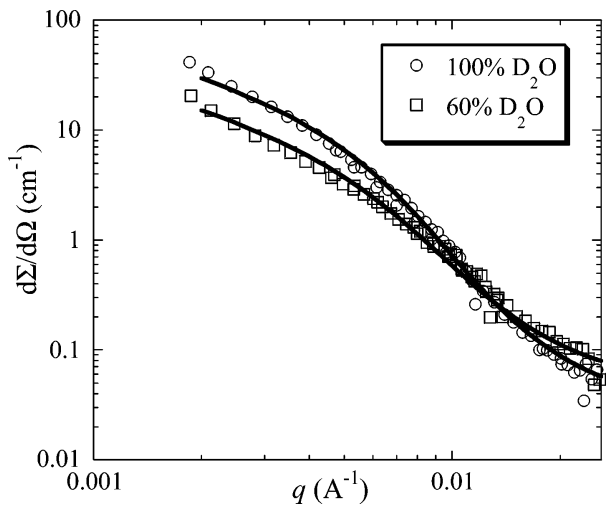

Figure 8. SANS patterns of the SWCNT/GA dispersions after subtraction of scattering from the polymer solution. Solid lines are the model fit to eq 3 .

more compact. As was mentioned before, the surface activity of GA is attributed to its minor component consisting of the arabinogalactan-protein complex. ${ }^{31}$ Thus, a high concentration of associated gum arabic macromolecules may be required to achieve a stable SWCNT dispersion.

For both systems, we are aware that these sets of parameters are not unique as there is a degree of uncertainty in the scattering length densities of the constituents. Yet, based on the chosen scattering length densities, the resulting parameters are physically reasonable and are self-consistent. Moreover, we believe that the general view of these large hybrid cylinders with a relative loose "swollen" interaction between the polymer and the nanotubes is valid and may be relevant to other dispersing polymer systems.

\section{Conclusion}

Stable dispersions of SWCNT in aqueous solution of GA and PSSty were achieved by a one-step procedure without harsh processes such as long sonication and ultracentrifugation. The dispersions were homogeneous having a dark inklike appearance and consisted of isolated nanotubes or very thin bundles, as was imaged by cryo-TEM. Both polymers, although differing in chemical structure and conformation, form the same structural association with the nanotubes. The adsorbed polymer forms a thick corona of solvent-swollen polymer coils around the nanotube core. These adsorbed large coils provide an effective steric barrier, as recently shown by simulation of poly(ethylene oxide) chains tethered to the SWCNT's surface. ${ }^{32}$ In the current case, steric stabilization is reinforced by electrostatic repulsion owing to charged groups distributed along the polymer. Hence, both polymers in this study exhibit qualitatively common scattering patterns of thick hybrid cylinders described by a modification of Pedersen's "block copolymer micelle" model. It is suggested than that long-term stable dispersions of SWCNTs can be accomplished by adsorption of diverse types of polymers as long as a significant steric barrier is introduced by thick envelopment of the nanotubes.

As a note concerning scattering analysis from nanotube dispersions, the cylindrical polymer/nanotube hybrid does not exhibit a $q^{-1}$ power law in the usually accessible $q$ range. Therefore, the absence of this power law is not necessarily an indication of poor dispersion, especially when polymers are involved. Complementary imaging of the dispersion morphology, such as by cryoTEM, is essential for a comprehensive evaluation.

To sum up, a quantitative characterization of SWCNT dispersions was presented, indicating the degree of debundling, the conformation of the attached polymer, and the stabilization mechanism. This characterization is relevant for subsequent processing of the dispersions. In particular, the large adsorbed coils may have a significant role as an interphase in SWCNT nanocomposites, linking the nanotube surface with the polymer matrix.

Acknowledgment. This project was supported by the Israel Science Foundation (Grant 26/03). Y.C. and Y.D. acknowledge support by the Jülich Neutrons for Europe program. We thank R. Yerushalmi-Rozen and I. Szleifer for helpful discussions and J. S. Pedersen for reviewing the manuscript.

\section{References and Notes}

(1) Vigolo, B.; Penicaud, A.; Coulon, C.; Sauder, C.; Pailler, R.; Journet, C.; Bernier, P.; Poulin, P. Science 2000, 290, 13311334.

(2) Moore, V. C.; Strano, M. S.; Haroz, E.; Hauge, R. H.; Smalley, R. E.; Schmidt, J.; Talmon, Y. Nano Lett. 2003, 3, 1379-1382.

(3) Islam, M. F.; Rojas, E.; Bergey, D. M.; Johnson, A. T.; Yodh, A. G. Nano Lett. 2003, 3, 269-273.

(4) Richard, C.; Blavoine, F.; Schultz, P.; Ebbesen, T. W. Mioskowski, C. Science 2003, 300, 775-778.

(5) Matarredona, O.; Rhoads, H.; Li, Z.; Harwell, J. H.; Balzano, L.; Resasco, D. E. J. Phys. Chem. B 2003, 107, 13357-13367.

(6) Yurekli, K.; Mitchell, C. A.; Krishnamoorti, R. J. Am. Chem. Soc. 2004, 126, 9902-9903.

(7) O’Connell, M. J.; Boul, P. J.; Ericson, L. M.; Huffman, C. B.; Wang, Y.; Haroz, E.; Kuper, C.; Tour, J.; Ausman, K. D.; Smalley, R. E. Chem. Phys. Lett. 2001, 342, 265-271.

(8) Zheng, M.; Jagota, A.; Strano, M. S.; Santos, A. P.; Barone, P.; Chou, S. G.; Diner, B. A.; Dresselhaus, M. S.; Mclean, R. S.; Onoa, G. B.; Samsonidze, G. G.; Semke, E. D.; Usrey, M.; Walls, D. J. Science 2003, 302, 1545-1548.

(9) Zorbas, V.; Ortiz-Acevedo, A.; Dalton, A. B.; Yoshida, M. M.; Dieckmann, G. R.; Draper, R. K.; Baughman, R. H.; JoseYacaman, M.; Musselman, I. H. J. Am. Chem. Soc. 2004, 126, $7222-7227$ 
(10) Bandyopadhyaya, R.; Nativ-Roth, E.; Regev, O.; YerushalmiRozen, R. Nano Lett. 2002, 2, 25-28.

(11) Shvartzman-Cohen, R.; Levi-Kalisman, Y.; Nativ-Roth, E.; Yerushalmi-Rozen, R. Langmuir 2004, 20, 6085-6088.

(12) Schaefer, D. W.; Zhao, J.; Brown, J. M.; Anderson, D. P.; Tomlin, D. Chem. Phys. Lett. 2003, 375, 369-375.

(13) Schaefer, D. W.; Brown, J. M.; Anderson, D. P.; Zhao, J.; Chokalingam, K.; Tomlin, D.; Ilavski, J. J. Appl. Crystallogr. 2003, 36, 553-557.

(14) Wang, H.; Zhou, W.; Ho, D. L.; Winey, K. I.; Fischer, J. E.; Glinka, C. J.; Hobbie E. K. Nano Lett., in press.

(15) Zhou, W.; Islam, M. F.; Wang, H.; Ho, D. L.; Yodh, A. G.; Winey, K. I.; Fischer, J. E. Chem. Phys. Lett. 2004, 384, 185189

(16) Wang, T. L.; Lee, H. M.; Kuo, P. L J. Appl. Polym. Sci. 2000, $78,592-602$.

(17) Permyakova, N.; Zheltonozhskaya, T.; Strilchyk, N.; Zagdanskaya, N.; Momot, L.; Syromyatnikov, V. Macromol. Symp. 2003, 203, 247-258.

(18) Garnier, G.; Duskova-Smrckova, M.; Vyhnalkova, R.; Van De Ven, T. G. M.; Revol, J. F. Langmuir 2000, 16, 3757-3763.

(19) Dror, Y.; Cohen, Y.; Yerushalmi-Rozen, R. J. Polym. Sci., Part B: Polym. Phys., in press.

(20) Bellare, J. R.; Davis, H. T.; Scriven, L. E.; Talmon, Y. J. Electron Microsc. Tech. 1988, 10, 87-111.
(21) Pedersen, J. S.; Gerstenberg, M. C. Macromolecules 1996, 29 , $1363-1365$

(22) Pedersen, J. S. Adv. Colloid Interface Sci. 1997, 70, 171210.

(23) Pedersen, J. S.; Svaneborg, C.; Almdal, K.; Hamley, I. W.; Young, R. N. Macromolecules 2003, 36, 416-433.

(24) Pedersen, J. S. J. Appl. Crystallogr. 2000, 33, 637-640.

(25) Hammouda, B. J. Polym. Sci., Part B: Polym. Phys. 1992 30, 1387-1390.

(26) Hammouda, B. Adv. Polym. Sci. 1993, 106, 87-133.

(27) Glatter, O.; Kratky, O. Small Angle X-Ray Scattering; Academic Press: London, 1982.

(28) Dror, Y.; Cohen, Y. Macromolecules, in press.

(29) Malardier-Jugroot, C.; van de Ven, T. G. M.; Whitehead, M. A. J. Mol. Struct. (THEOCHEM) 2004, 679, 171-177.

(30) Connolly, S.; Fenyo, J. C.; Vandervelde, M. C. Carbohydr. Polym. 1988, 8, 23-32.

(31) Randall, R. C.; Phillips, G. O.; Williams, P. A. Food Hydrocolloids 1988, 2, 131-140.

(32) Shvartzman-Cohen, R.; Nativ-Roth, E.; Baskaran, E.; LeviKalisman, Y.; Szleifer, I.; Yerushalmi-Rozen, R. J. Am. Chem. Soc. 2004, 126, 14850-14857.

MA0503615 